\title{
SAÚDE E BEM-ESTAR DE CRIANÇAS EM IDADE ESCOLAR
}

\author{
School aged children health and welfare
}

\author{
Salud y bienestar de los niños en edad escolar
}

\section{RESUMO}

A percepção que as crianças têm sobre a sua própria saúde é um importante indicador para a definição de estratégias de promoção de saúde infantil. Este estudo correlacional analisa a percepção de saúde de uma amostra probabilística acidental de 216 crianças portuguesas do ensino básico. 0 questionário de recolha de dados (CHIP-CE) revelou boas propriedades psicométricas (alfa $=0,84)$. Os resultados indicam médias e desvios-padrão de cinco fatores: Conforto $(4,34 ; 0,45)$; Evitamento de risco $(4,18$; $0,53)$; Satisfação $(4,03 ; 0,56)$; Resiliência $(3,75 ; 0,60)$; Realização $(3,75 ; 0,60)$. Resiliência e evitamento de risco são fatores com valores mais baixos. A dificuldade em realizar os trabalhos de casa e conviver com os colegas é indicadora da dimensão resiliência mais problemática. Os resultados permitem descrever o perfil de saúde e planejar uma intervenção de educação para a saúde das crianças, em contexto escolar, especialmente na área do relacionamento e motivação para o estudo.

Palavras-chave: Bem-Estar da Criança. Perfil de Saúde. Saúde Escolar

\begin{abstract}
The perception that children have about their own health is an important indicator for devising strategies to promote child health. This co relational study analyzes the health perceptions of an accidental probability sample of 216 Portuguese children during basic education. The data collection questionnaire (CHIP-CE) showed good psychometric properties (alpha $=0.84$ ). The results show the following averages and standard deviations for five factors: Comfort (4.34, 0.45), Risk avoidance $(4.18,0.53)$, Satisfaction $(4.03,0.56)$, Resilience $(3.75 ; 0.60)$, and Achievement $(3.75,0.60)$. Resilience and Risk avoidance are factors with lower values. Difficulties in doing the homework and in socializing with peers identify the problematic indicators of the resilience scale. The results allow us to describe health profiles and health plan education interventions for school children, especially regarding the relationships and motivation for studying.
\end{abstract}

Keywords: Child Welfare. Health Profile. School Health

\section{Resumen}

La percepción que los niños tienen sobre su propia salud es un importante indicador para la definición de estrategias de promoción de salud infantil. Este estudio correlacional analiza la percepción de salud de una muestra probabilística accidental de 216 niños portugueses de escolaridad básica. El cuestionario de recolección de datos (CHIP-CE) reveló buenas propiedades psicométricas (alfa $=0,84)$. Los resultados indican medias y desvíos estandarizados de cinco factores: Comodidad (4,34; 0,45); Evitación de riesgo (4.18; 0,53); Satisfacción (4,03; $0,56)$; Resiliencia $(3,75 ; 0,60)$; Realización $(3,75 ; 0,60)$. Resiliencia y evitación de riesgo son los factores con valores más bajos. La dificultad en realizar los deberes y convivir con los compañeros son indicadores de la dimensión de resiliencia más problemáticos. Los resultados permiten describir el perfil de salud y planear una intervención de educación para la salud de los niños, en contexto escolar, especialmente en el área del relacionamiento y motivación para el estudio.

Palabras clave: Bienestar del Niño. Perfil de Salud. Salud Escolar

'Especialista em Enfermagem de Saúde Mental e Psiquiátrica, Mestre em Ciências da Educação, Hospital da Universidade Coimbra, Coimbra, Zona Centro, Portugal Email:isanoronha@sapo.pt²Professor Coordenador com Agregação, Coordenador Científico da Unidade de Investigação da Escola Superior de Enfermagem de Coimbra, Coimbra, Zona Centro, Portugal. E-mail: demar7@gmail.com 


\section{INTRODUÇÃO}

0 conceito de saúde é complexo, multidensional, multideterminado e subjetivo, com representações diferentes para cada pessoa, em função do seu modo particular de perceber o mundo. No âmbito do processo de desenvolvimento humano, a percepção de saúde depende do projeto de vida de cada um, do seu sentido de felicidade e do seu esforço para compreender e respeitar o Universo. ${ }^{1} 0$ valor subjetivo de saúde é um indicador importante para avaliação de saúde, em qualquer fase do desenvolvimento humano. Durante muito tempo, permaneceu a tendência de valorizar a opinião que os pais têm sobre a saúde dos filhos, mais do que o ponto de vista das próprias crianças. No entanto, a investigação passou a valorizar a percepção da criança, sobre o seu estado de saúde. ${ }^{2,3} \mathrm{~A}$ Psicologia defende que as crianças, entre os oito e os onze anos, já possuem habilidades cognitivas que as capacitam para diferenciar as suas ideias das de outras pessoas e de as expressar verbalmente. ${ }^{4,5}$ Estes estudos estão em consonância com as orientaç̃̃es da Organização Mundial de Saúde. A avaliação da saúde percebida tem evidenciado que as crianças em idade escolar tendem a revelar uma percepção positiva da sua saúde. .- $^{6-}$ ${ }^{8}$ Também associam a saúde ao desejo de liberdade, visto que esta the permite realizar atividades que não conseguem executar quando doentes. ${ }^{9}$ A capacidade perceptiva das crianças, em relação ao seu estado de saúde e bem-estar, permite utilizar instrumentos de medida que ajudam a efetuar o diagnóstico de necessidades específicas de saúde. ${ }^{10}$ É importante o diagnóstico atempado de problemas e necessidades de saúde, para a prevenção e proteção em situações de risco, sobretudo quando a criança é vitima de situações de iniquidade e violência, as quais representam uma dolorosa experiência para ela, cuja superação depende de fortes fatores de protecção. ${ }^{11} 0$ Diagnóstico de necessidades de saúde é um elemento fulcral de ação estratégica dos serviços de saúde e das escolas promotoras de saúde, para a prevenção de situações que estão muitas vezes na origem do insucesso escolar, abandono e exclusão. 12,13 Muitas das intervenções de educação para a saúde são postas em prática sem diagnóstico prévio das necessidades de saúde espećíicas das crianças, elaboradas com a imposição do conhecimento dos profissionais de saúde e de educação, por vezes com estratégias pouco adequadas às capacidades de aprendizagem de cada criança.

Na Unidade de Investigação em Ciências da Saúde: Enfermagem, no contexto da linha de investigação "a formação de profissionais de saúde e estratégias de educação para a saúde", está em desenvolvimento o projeto de investigação "Promoção de saúde e educação para a saúde de crianças em contexto escolar", o qual aplica o Método Eduterapêutico ${ }^{14}$ que parte dos seguintes pressupostos: a educação para a saúde infantil é uma prioridade do Plano Nacional de Saúde; as crianças em idade escolar (entre os oito e os doze anos) estão em fase de desenvolvimento favorável à promoção de estratégias criativas de educação para a saúde; a intervenção mais precoce possível na cadeia educativa ajuda a construção de atitudes facilitadoras para ter melhor saúde e também na aquisição de comportamentos saudáveis; a Escola é um setting adequado à promoção da educação para a saúde, sobretudo quando se envolvem, a criança, os pais e professores; as intervenções de educação para a saúde devem ser planeadas a partir das necessidades específicas de saúde das crianças e adequadas às suas características específicas pessoais e de contexto. Este estudo está inserido no referido projeto, tem por base a mesma problemática e recorre a instrumentos já validados para a população portuguesa, orientados para o diagnóstico do perfil de saúde e bem-estar das crianças com posterior intervenção em contexto escolar.

\section{METODOLOGIA}

\section{Tipo de estudo:}

\section{Descritivo e correlacional}

\section{Questão}

Quais as características do perfil de saúde percebido pelas crianças em idade escolar $8-12$ anos?

\section{Hipóteses}

A percepção de saúde é diferente entre meninos e meninas.Verifica-se correlação entre a idade e a percepção de saúde e bem-estar das crianças..A percepção de saúde e bemestar é diferente entre as crianças em monodocência e as crianças em pluridocência. A percepção de saúde e bem-estar difere consoante 0 ano de escolaridade.

\section{População/ Amostra}

A população-alvo integra 261 crianças com idades compreendidas entre 8 e 12 anos do ensino básico de duas instituições pertencentes ao concelho de Coimbra. Utilizou-se a técnica de amostragem não probabilística acidental e obtiveram-se respostas de 216 crianças que estavam presentes em sala de aula no momento da colheita de dados, o que representa $82,75 \%$ da população-alvo.

\section{Instrumentos}

0 instrumento de medida utilizado ${ }^{10}$ foi traduzido para português por uma equipe da Unidade de Investigação da Escola Superior de Enfermagem de Coimbra, no contexto do Projeto de Promoção de Educação para a Saúde de Crianças em Contexto Escolar. Relativamente ao total da 
escala, são revelados valores de correlação do item com o total da escala corrigida que oscilam entre 0,20 e 0,64, com um alfa total de $0,84,0$ que atesta a favor da fiabilidade do instrumento. Estes resultados são semelhantes aos indicados originalmente pelos autores, com valores de alfa que variavam entre 0,70 e 0,80. Estudamos também a consistência interna das dimensões da escala através do coeficiente alpha de Cronbach: satisfação $(a=0,84)$; conforto $(a=0,78)$; resiliência $(a=0,70)$; evitamento de risco $(a=0,71)$; realização $(a=0,78)$.

\section{Procedimentos Éticos}

Para a realização da investigação foram desenvolvidas estratégias no sentido de seguir alguns dos princípios éticos que são inerentes aos trabalhos de investigação. Assim, para efetuar a recolha de dados, recorremos à autorização formal da Comissão Nacional de proteção de dados, Direção Regional de Educação do Centro e Direção das Escolas. A fim de assegurar o consentimento informado, foram contatados os encarregados de educação das crianças, que participaram dando-lhe conhecimento dos objetivos do estudo e garantindo-lhes confidencialidade dos dados obtidos. Neste processo, tivemos a colaboração dos diretores de turma, que enviaram e recolheram as autorizações.

\section{RESULTADOS E DISCUSSÕES}

\section{Caracterização da amostra}

A amostra é constituída por 216 crianças do ensino básico, sendo $55,1 \%$ do sexo masculino e $44,9 \%$ do sexo feminino. A média das idades é de 10,15 anos, 42,6\% entre 11 e 12 anos, e 36,6\% entre 10 e 11 anos. 43,6\% das crianças frequentam o $5^{\circ}$ ano de escolaridade e $32,4 \%$ frequentam o $6^{\circ}$ ano.

\section{Resultados em cada um dos fatores da escala de perfil de saúde}

Analisando os resultados apresentados no Quadro 1, podemos verificar que, na dimensão "satisfação", os resultados variaram entre 2,44 e 5,00, sendo a média de 4,03 com desvio-padrão de 0,56. Metade das crianças apresentou resultados acima de 4,06, e 57,9\% tiveram resultados entre 4 e 5 . Para a dimensão "conforto" observamos resultados compreendidos entre 2,83 e 5,00, situando-se a média nos 4,34 com desvio-padrão de 0,45 . Com resultados entre 4 e 5 observamos $82,4 \%$ das crianças e $50,00 \%$ apresentaram valores acima de 4,33.Verificamos que, na dimensão "resiliência", 47,2\% das crianças apresentaram resultados compreendidos entre 4 e 5 e igual percentagem apresentou resultados entre 3 e 4 pontos. Os escores variaram entre 2,00 e 5,00 , metade estavam acima de 3,88 e a média era de 3,89 com desvio-padrão de 0,56. Em termos da dimensão "evitamento de risco", verificamos que $69,9 \%$ das crianças apresentaram resultados entre 4 e 5 pontos e $50,00 \%$ obtiveram pontuações acima de 4,25 . Os valores situaram-se entre 2,63 e 5,00, sendo a sua média de 4,18 com desvio-padrão de 0,53 . Para a dimensão "realização" observamos resultados compreendidos entre 2,25 e 5,00, e a maioria das crianças, concretamente $51,4 \%$, obteve valores entre 3 e 4.0 valor médio foi de 3,75 com desvio-padrão de 0,60.

Quadro 1 - Medidas descritivas observadas em cada dimensão

\begin{tabular}{|l|c|c|c|c|c|}
\hline Dimensão & $\bar{x}$ & $M d$ & $s$ & $x_{\min }$ & $x_{\text {máx }}$ \\
\hline Satisfação & 4,03 & 4,06 & 0,56 & 2,44 & 5,00 \\
\hline Conforto & 4,34 & 4,33 & 0,45 & 2,83 & 5,00 \\
\hline Resiliência & 3,89 & 3,88 & 0,56 & 2,00 & 5,00 \\
\hline Evitamento de risco & 4,18 & 4,25 & 0,53 & 2,63 & 5,00 \\
\hline Realização & 3,75 & 3,75 & 0,60 & 2,25 & 5,00 \\
\hline
\end{tabular}

\section{Relação entre perfil de saúde e sexo}

De acordo com o Quadro 2, a comparação dos dados de saúde percebida em função do sexo da criança, revela que apenas na dimensão "evitamento de risco" existe diferença estatisticamente significativa $(p=0,004)$, ou seja, o perfil de saúde difere significativamente das crianças do masculino para as do sexo feminino em termos de 
evitamento de risco. Comparando os valores das medidas de tendência central, podemos ainda verificar que, nesta dimensão, as crianças do sexo feminino evidenciam melhor resultado.

Quadro 2 - Estatísticas do teste Mann-Whitney U relativas às diferentes dimensões da saúde percebida segundo o sexo

\begin{tabular}{|c|c|c|c|c|c|c|}
\hline $\begin{array}{c}\text { Perfil de saúde } \\
\text { Sexo }\end{array}$ & $n$ & $\bar{x}_{\text {ord }}$ & $\bar{x}$ & Md & 2 & $p$ \\
\hline Satisfação & & & & & $-0,159$ & 0,874 \\
\hline Masculino & 119 & 107,89 & 4,01 & 4,00 & & \\
\hline Femin ino & 97 & 109,25 & 4,04 & 4,11 & & \\
\hline Conforto & & & & & $-1,792$ & 0,073 \\
\hline Masculino & 119 & 115,37 & 4,38 & 4,42 & & \\
\hline Feminino & 97 & 100,08 & 4,28 & 4,33 & & \\
\hline Resiliência & & & & & $-0,939$ & 0,348 \\
\hline Masculino & 119 & 104,90 & 3,85 & 3,88 & & \\
\hline Feminino & 97 & 112,91 & 3,94 & 3,88 & & \\
\hline Evitamento de risco & & & & & $-2,857$ & 0,004 \\
\hline Masculino & 119 & 97,57 & 4,08 & 4,12 & & \\
\hline Femin ino & 97 & 121,91 & 4,30 & 4,38 & & \\
\hline Realização & & & & & $-0,695$ & 0,487 \\
\hline Masculino & 119 & 105,84 & 3,73 & 3,62 & & \\
\hline Feminino & 97 & 111,77 & 3,78 & 3,88 & & \\
\hline
\end{tabular}

\section{Relação entre percepção de saúde e idade}

Como podemos constatar no Quadro 3, as correlações são estatisticamente significativas ao nível das dimensões "satisfação" ( $p=0,039)$, "conforto" $(p=0,039)$ e "realização" ( $p<0,001)$. Este fato permite-nos concluir que existe relação entre as duas variáveis nestas três dimensões. 0 fato de o coeficiente de correlação apresentar valor negativo na "satisfação" e na "realização" e positivo no "conforto", permitenos ainda afirmar que as crianças mais velhas tendem a evidenciar pior perfil de saúde em termos de satisfação e realização e melhor perfil de saúde ao nível de conforto.

Quadro 3 - Estatísticas do teste de correlação $r_{\mathrm{s}}$ da idade com cada uma das dimensões de saúde percebida

\begin{tabular}{|l|c|c|}
\hline \multirow{2}{*}{ Dimensão } & \multicolumn{2}{|c|}{ Idade $(n=216)$} \\
\cline { 2 - 3 } & $r_{s}$ & $p$ \\
\hline Satisfação & $-0,14$ & 0,039 \\
\hline Conforto & $+0,14$ & 0,039 \\
\hline Resiliência & 0,01 & 0,993 \\
\hline Evitamento de risco & $-0,08$ & 0,232 \\
\hline Realização & $-0,34$ & $<0,001$ \\
\hline
\end{tabular}




\section{Relação entre o perfil de saúde e 0 tempo de escolaridade}

Um estudo idêntico permitiu avaliar a relação entre o perfil de saúde e 0 ano de escolaridade que as crianças frequentam. Os resultados apresentados no Quadro 4 permitem-nos constatar que as correlações também são estatisticamente significativas ao nível das dimensões "satisfação" ( $p=0,044)$, "conforto" $(p=0,049)$ e "realização" ( $p<0,001)$. Sendo o coeficiente de correlação negativo na "satisfação" e na "realização" e positivo no "confor to", podemos afirmar que as crianças com maior escolaridade tendem a evidenciar pior perfil de saúde em termos de satisfação e realização e melhor perfil de saúde ao nível de conforto. Estas conclusões coincidem com as que tiramos a partir dos resultados do estudo da correlação com a idade. Esta situação não é de estranhar dado que as crianças com mais escolaridade tendem a ser mais velhas e vice-versa; ou seja, entre 0 ano de escolaridade e a idade existe uma correlação muito forte.

Quadro 4-Correlacão entre o ano escolar e as diferentes dimensões

\begin{tabular}{|l|c|c|}
\hline \multirow{2}{*}{ Dimensäo } & \multicolumn{2}{|c|}{ Ano de escolaridade } \\
& \multicolumn{2}{|c|}{$(n=216)$} \\
\cline { 2 - 3 } & $r_{s}$ & $p$ \\
\hline Satisfação & $-0,14$ & $\mathbf{0 , 0 4 4}$ \\
\hline Conforto & $+0,13$ & $\mathbf{0 , 0 4 9}$ \\
\hline Resiliência & $-0,01$ & 0,963 \\
\hline Evitamento de risco & $-0,01$ & 0,947 \\
\hline Realização & $-0,27$ & $<0,001$ \\
\hline
\end{tabular}

Relação entre o perfil de saúde e o tipo de docência

Comparando os dados de saúde percebida, Quadro 5, em função do tipo de docência, verificamos a existência de diferenças estatisticamente significativas em termos de "conforto" ( $p<0,001)$ e de "realização" ( $p=0,006)$. Comparando os valores das medidas de tendência central, verificamos que as crianças que se encontram na situação de monodocência revelam pior perfil de saúde ao nível do "conforto" e melhor perfil de saúde em termos de realização.

Quadro 5 - Estatísticas do teste Mann-Whitney U relativas ás diferentes dimensões da saúde percebida e tipo de docência

\begin{tabular}{|c|c|c|c|c|c|c|}
\hline $\begin{array}{l}\text { Saúde Percebida } \\
\qquad \text { Tipo de docênci }\end{array}$ & $\mathrm{n}$ & $\bar{x}_{\text {ord }}$ & $\bar{x}$ & $\mathrm{Md}$ & $\mathrm{z}$ & $\mathrm{p}$ \\
\hline Satisfaçăo & & & & & $-0,589$ & 0,556 \\
\hline Monodocência & 48 & 113,18 & 4,08 & 4,17 & & \\
\hline Pluridocêcnia & 168 & 107,16 & 4,01 & 4,00 & & \\
\hline Conforto & & & & & $-3,953$ & $<0,001$ \\
\hline Monodocência & 48 & 77,11 & 4,08 & 4,17 & & \\
\hline Plunidocêcnia & 168 & 117,47 & 4,41 & 4,46 & & \\
\hline Resiliência & & & & & $-0,400$ & 0,689 \\
\hline Monodocência & 48 & 105,32 & 3,86 & 3,88 & & \\
\hline Plunidocêcnia & 168 & 109,41 & 3,90 & 3,87 & & \\
\hline Evitamento de risco & & & & & $-1,526$ & 0,127 \\
\hline Monodocência & 48 & 96,40 & 4,06 & 4,19 & & \\
\hline Plunidocêcnia & 168 & 111,96 & 4,22 & 4,25 & & \\
\hline Realização & & & & & $-2,739$ & 0,006 \\
\hline Monodocência & 48 & 130,24 & 3,96 & 3,88 & & \\
\hline Pluridocêcnia & 168 & 102,29 & 3,69 & 3,62 & & \\
\hline
\end{tabular}




\section{DISCUSSÃO}

Em resposta à questão de investigação "quais as características do perfil de saúde das crianças" em estudo, verificamos que as respostas das crianças apontam para um nível razoável e bom, sendo melhor nas dimensões de conforto e evitamento de risco e um pouco mais baixo em termos de realização e resiliência. Estes resultados vêm de encontro à opinião de alguns autores, ${ }^{6-8}$ que referem que as crianças na infância, de uma forma geral, têm uma boa percepção da sua saúde, excluindo as situações de doenças típicas da infância. Nos últimos anos, tem aumentado o número de casos de doença oncológica em crianças, bem como o número de situações de bullying em contexto escolar, que, de acordo com a literatura, tem influência nos indicadores de saúde, resiliência e realização. Os resultados indicam que as crianças do sexo feminino evidenciam uma melhor percepção de saúde que as do sexo masculino $(p=0,004)$, em coerência com os resultados de investigações relevantes..$^{10}$ Esta atitude mais preventiva das meninas tende a observar-se ao longo das diferentes fases do desenvolvimento. As diferenças de gênero estão relacionadas com o desenvolvimento físico ${ }^{6}$ a forma como as meninas brincam, as quais, em geral, têm menos força física e tendem a evitar atividades mais perigosas. Verificou-se que as crianças com mais idade revelam, por um lado, melhores resultados no conforto $(r=0,14, p=0,039)$ e, por outro lado, resultados mais baixos na satisfação $(r=-0,14, p=-0,039)$ e na realização $(r=-0,34, p=0,001)$. Estes resultados vem corroborar a opinião segundo a qual as crianças no estádio das operações concretas refletem sobre os seus verdadeiros sentimentos $e$ percebem a importância das mudanças. ${ }^{4}$ As crianças já são capazes de perceber os acontecimentos a partir do ponto de vista dos outros, evidenciando capacidades de resiliência e auto realização. ${ }^{3}$ Seguindo a tendência dos resultados em relação à idade, também em relação ao tempo de escolaridade, observase que as crianças que estão há mais tempo na escola revelam menos satisfação $(r=-0,14, p=0,044)$ e menos realização $(r=-$ $0,27, p=0,001)$, e melhor percepção de conforto $(r=0,13$, $p=0,049)$. A relação da criança com a escola nem sempre é uma situação paćifica, de acordo com os estudos sobre insucesso e abandono escolar, indisciplina, disrupção, bullying, sentimento de insatisfação com a escola e estudo; além disso, o sentimento de incapacidade para resistir aos desafios tende a aumentar com o tempo. Uma atenção especial deve ser dada às vivências de experiências de violência, que afetam o desenvolvimento integral da criança. ${ }^{11}$ Ao testar a hipótese da relação entre a situação escolar de monodocêncoa ou pluridocência em que as crianças se encontram verificou-se que, na dimensão conforto, a diferença é significativa $(z=-3,953, p=0,001)$, com uma média mais elevada para as crianças em pluridocência. No entanto, o sentimento de realização tende a ser melhor em situação de monodocência $(z=2,739, p=0,006)$. Poderemos pensar que o sentimento de conforto tende a ser mais valorizado à medida que a crianças interagem com os seus pares, identificando-se com eles e recebendo o seu apoio de pertença ao grupo. ${ }^{15,4}$ Quando analisamos os componentes da variável latente, verificamos que, na dimensão satisfação, os indicadores mais problemáticos referem-se às relações pais/filhos e, na dimensão resiliência, os indicadores mais problemáticos são a dificuldade em realizar trabalhos de casa e conviver com colegas.

\section{CONCLUSÃO}

De acordo com os objetivos, descreveram-se as características sociodemográficas das crianças e definiu-se um perfil de saúde percebida em função dos fatores prevenção de risco, resiliência, conforto, satisfação, realização. 0 diagnóstico do perfil de saúde e bem-estar das crianças pôs em evidência os indicadores e as dimensões em que as crianças revelaram uma percepção mais negativa de saúde. Os resultados ajudam a planear estratégias de intervenção em contexto escolar, para empoderamento, apoio relacional de ajuda e promoção de autoestima das crianças. A ajuda deve orientar-se para a promoção da resiliência e no sentido de realização das crianças, considerando o seu nível de desenvolvimento e o contexto em que se encontram. Há crianças que necessitam de ajuda para enfrentar as dificuldades do cotidiano familiar, social e escolar. Algumas crianças revelaram baixos recursos de capital social, fundamental para estimular o interesse pelas atividades escolares e relacionamento com os outros. Os resultados indicam a necessidade de maior articulação entre todos os intervenientes e responsáveis pela saúde escolar, ajudando as crianças mais desfavorecidas na ação protetora de risco, na integração social e na motivação para o estudo.

\section{REFERÊNCIAS}

1. Rodrigues, M., Pereira, A., Barroso, T. Educação para a saúde: Formação Pedagógica de Educadores de saúde. Coimbra: Formasau-Formação e saúde, Lda; 2005

2. Ferreira, P. (1992). Medição do estado de saúde em crianças. Saúde Infantil, 14: 157-163.

3. Sylva, K., Lunt, I. Iniciação ao desenvolvimento da criança, São Paulo: Martins Fontes; 1994

4. Selmon, R. The growth of interpersonal understanding. New York: Academie Press; 1980 
5. Veríssimo, M. A experiência de hospitalização explicada pela própria criança. Revista da Escola de Enfermagem de São Paulo, 1981; 25 (2): $153-168$.

6. Bee, H. A criança em desenvolvimento. $7^{\mathrm{a}}$ ed. Porto Alegre, Artes Médicas; 2003

7. Gesell A. A criança dos 0 aos 5 anos - São Paulo: Martins Fontes; 1985 8. Papalia D, Olds, S. Desenvolvimento Humano. $7^{\text {a }}$ ed., Portalegre: Artes Médicas; 2000

9. Moreira P, Dupas, G. Significado de saúde e doença na percepção da criança. Revista Latina - Americana de Enfermagem, Brasil, 2003 Nov. I Dez: 757 - 762

10. Riley A, Forrest C, Rebok G, Starfield B, Green B, Robertson J, Friello P - The Child Report Form of the CHIP-Child Edition. Medical Care. 2004; $42(3): 221-231$

11. Pierantoni, Lúcia Martins de Magalhães; Cabral, Ivone Evangelista Crianças em situação de violência de um ambulatório do Rio de Janeiro: conhecendo o seu perfil. Escola Anna Nery Revista de Enfermagem. 2009 Out-Dez; 13 (4): 699-707

12. Ministério da Saúde - Programa Nacional de saúde Escolar. Lisboa: DGS, 2006

13. Observatório português dos sistemas de saúde: Desafios em tempos de crise: relatório da primavera 2010. Lisboa: Mar da Palavra; 2010

14. Rodrigues $M$, Hawrylack $M$ - 0 Metodo Eduterapêutico aplicado à educação para a saúde de crianças em contexto escolar. Revista de enfermagem Referência. 2007 Dez; II Série (5): 69-76

15. Erikson, E. Infância e sociedade. $2^{\mathrm{a}}$ ed. Nova York; 1976 